\title{
Quick Performance Assessment of Improved Nyquist Pulses
}

\author{
Nicolae Dumitru Alexandru and Felix Diaconu \\ Department of Telecommunications, "Gheorghe Asachi” Technical University of Iaşi, Bd. Carol I, No. 11A, 700506 Iaşi, Romania \\ Correspondence should be addressed to Felix Diaconu; fdiaconu@etti.tuiasi.ro
}

Received 7 July 2016; Accepted 20 September 2016; Published 11 January 2017

Academic Editor: Javier Del Ser

Copyright (C) 2017 N. D. Alexandru and F. Diaconu. This is an open access article distributed under the Creative Commons Attribution License, which permits unrestricted use, distribution, and reproduction in any medium, provided the original work is properly cited.

\begin{abstract}
An explanation is proposed for the improved behavior of the improved Nyquist pulses with an asymptotic decay rate of $t^{-2}$ when sampled with a timing offset. Three figures of merit that indicate the energy distribution into the sidelobes of the time response and allow a quick assessment of their performance in terms of error probability when the impulse response is sampled with a timing error have been proposed and verified on several improved Nyquist pulses reported in the literature. In order to check the validity of the proposed figures of merit a novel family of Nyquist pulses denoted as power sine was introduced. Using the proposed approach the design process was expedited as the volume of necessary calculations was significantly decreased. To explain the difference in close pulse performance a figure of merit based on limited ISI distortion was introduced.
\end{abstract}

\section{Introduction}

In the design of a Nyquist filter the robustness to timing jitter is a prime factor. A solution is to redistribute the tail energy of impulse response by diminishing the size of the largest sidelobe and in turn increase the size of the subsequent sidelobes. The result is a decrease of the intersymbol interference (ISI), which manifests itself in a decrease of the error rate when the impulse response is sampled at the receiver's site with a timing error. This approach was first proposed and used in [1].

Beaulieau et al. [1,2] demonstrated that a Nyquist pulse that decays asymptotically as $t^{-2}$ performs better than a pulse with an asymptotic decay rate (ADR) of $t^{-3}$, such as the standard raised cosine ( $\mathrm{RC}$ or rcos) pulse, in terms of error probability when sampled with a timing offset. In practice the pulse is generated digitally using a truncated version of the impulse response of the Nyquist filter and is digitally filtered at the receiver, using an adapted filter to its truncated version, which introduces spectral re-growth.

The new pulses with a slower $\operatorname{ADR}\left(t^{-2}\right.$ versus $\left.t^{-3}\right)$ require a bigger number of taps when implemented digitally in a finite impulse response (FIR) structure, as compared with the RC pulse. Stated in another way, for a given truncated length, the spectral regrowth of a slowly decaying pulse is worse than that of a fast decaying pulse. In order to obtain comparable performance in terms of spectral regrowth, one should increase the number of filter taps, resulting in bigger latency and implementation costs.

In some applications where the latency is not critical these disadvantages are counteracted by the fact that one obtains increased performance in terms of lower bit error rate (BER).

Several researchers embraced this idea known as improved Nyquist filter (INF) or pulse and produced on heuristic bases novel pulses that performed better than the ones previously reported [3-11]. However, the mechanism behind the improved performance of Nyquist pulses was not completely understood so far. In the sequel we investigated the behavior of several improved Nyquist pulses that decay asymptotically as $t^{-2}$ and $t^{-3}$ and proposed three figures of merit to quickly assess their performance in terms of error probability when the impulse response is sampled with a timing error.

Also, a novel family of Nyquist pulses, denoted as power sine was introduced in order to check the validity of the approach to expedite the design process.

\section{Fractional Energy}

To make the tails of the impulse response less damped, that is, to increase its oscillatory feature it is necessary to transfer an amount of energy from the lower frequency part of the transition region $[B(1-\alpha), B]$ into the higher one $[B, B(1+\alpha)]$ 
TABLE 1: Analytic expressions of fractional energy for several Nyquist pulses.

\begin{tabular}{|c|c|c|}
\hline Pulse & $E_{s}$ & $E_{l}$ \\
\hline $\mathrm{rcos}$ & $\frac{\alpha B}{2 \pi}(\pi-2)$ & $\frac{\alpha B}{2 \pi}(\pi+2)$ \\
\hline fsech & $\frac{\alpha B}{2 \pi}\left(1-\frac{\pi}{3 \cdot a \cosh (2)}\right)$ & $\frac{\alpha B \pi}{3 \cdot a \cosh (2)}$ \\
\hline fexp & $\alpha B-\frac{\alpha B}{\log (4)}$ & $\frac{\alpha B}{\log (4)}$ \\
\hline farceerb $(R-1)$ & $2 \pi \alpha / 3-\alpha \log (2+\sqrt{3})$ & $2 \pi \alpha / 3-\alpha \log (2+\sqrt{3})$ \\
\hline Power $(B=1)$ & $\begin{array}{c}2 \cdot a \cosh (2) \\
\frac{\alpha}{2(1+\beta)}\end{array}$ & $\begin{array}{c}2 \cdot a \cosh (2) \\
\alpha-\frac{\alpha}{2(1+\beta)}\end{array}$ \\
\hline Poly $(\alpha, b, c, d)$ & $\frac{\alpha[20 b+15(c+8)+9 d]}{480}$ & $-\frac{\alpha[20 b+15 c+9(d-40)]}{480}$ \\
\hline
\end{tabular}

TABLE 2: Analytic expressions of slope at $t / T=1$ for several Nyquist pulses.

\begin{tabular}{lc}
\hline Pulse & Slope at $t / T=1$ \\
\hline rcos & $-\frac{\cos (\alpha \pi)}{1-4 \alpha^{2}}$ \\
fexp & $\frac{\ln (2)[\ln (2)-\cos (\alpha \pi) \ln (4)-2 \alpha \pi \sin (\alpha \pi)]}{(\alpha \pi)^{2}+[\ln (2)]^{2}}$ \\
Poly $(b, c, d)$ & $-\frac{24 d-4[2 b+3(c+d)] x^{2}+8\left(b x^{2}-3 d\right) \cos (x)+x\left[12 c+(8+4 d+2 c+d) x^{2}\right] \sin (x)}{8 x^{4}}$ \\
Power & $-1+\frac{x^{2}{ }_{1} F_{2}\left[\{1\},\{3 / 2+\beta / 2,2+\beta / 2\},-(1 / 4) x^{2}\right]}{2+3 \beta+\beta^{2}}$ \\
\hline
\end{tabular}

$[7,9]$. The parameter $\alpha$ is known as excess bandwidth or rolloff factor.

his is to say the frequency characteristic should be concave in the $[B(1-\alpha), B]$ range and in view of odd-symmetry convex in the $[B, B(1+\alpha)]$ range. Otherwise stated, we wish to decrease the fractional energy contained in the lower frequency band $[B(1-\alpha), B]$ of the time-unlimited Nyquist pulse by transferring some energy into the higher frequency band $[B, B(1+\alpha)]$, that is, to increase the fractional energy contained in the frequency band $[B, B(1+\alpha)]$ up to some level.

As a figure of merit regarding the efficiency of this transfer we propose to take the ratio of the pulse energy contained either in the frequency interval $[B, B(1+\alpha)]$ or $[B(1-\alpha), B]$ to the total energy contained in the frequency interval $[0, B(1+\alpha)]$, by taking into account only the positive frequency components. We will denote it as fractional energy $E_{s}$ and $E_{l}$, respectively,

$$
E_{s}=\int_{B}^{B(1+\alpha)}|H(f)| d f .
$$

Also,

$$
E_{l}=\int_{B(1-\alpha)}^{B}|H(f)| d f .
$$

For a Nyquist filter the energy carried in the frequency interval $[0, B(1-\alpha)]$ is obviously $(1-\alpha)$ for $B=1$. So,

$$
E_{l}+E_{s}=\alpha .
$$

This is justified by the fact that $H(f)$ has unit energy, as this is a Nyquist filter.
A higher value of the fractional energy $E_{s}$ denotes that more energy is transferred within the transition band of the filter from $[B(1-\alpha), B]$ to the $[B, B(1+\alpha)]$ frequency interval.

A similar approach was found in [12] where a similar metric was used. This is calculated by taking the ratio of the root-Nyquist pulse energy between $1 /(2 T)$ and infinity, to its total energy.

Table 1 reports the analytic expressions of fractional energy for several Nyquist pulses that are mathematically tractable. For other pulses one must use numerical integration for given values of excess bandwidth $\alpha$.

\section{Slope of the Time Response at Its First Zero Crossing}

The improved performance of the new Nyquist pulse in [1] when sampled with a time offset is explained based on the fact that the magnitudes of the two largest sidelobes [1] are smaller than the magnitudes of the RC pulse. Also in [7] one shows that the new Nyquist pulses exhibit a more pronounced decrease in the amplitudes of the two largest sidelobes, which accounts for their improved robustness to error probabilities.

Based on these assertions two figures of merit are proposed.

The first one is the slope of the time response evaluated at its first zero crossing, $t / T=1$. Obviously, the higher the slope of time response evaluated at $t / T=1$, the higher the magnitude of the first sidelobe is expected to be.

Several pulses, such as RC, BTRC (fexp) [1], Poly [5], and Power [8], have time responses given by closed-form 
TABLE 3: Analytic expressions of second derivative of time response at $t / T=1$ for several Nyquist pulses.

\begin{tabular}{|c|c|}
\hline Pulse & Derivative of slope at $t / T=1$ \\
\hline \multirow{2}{*}{$\operatorname{rcos}$} & $2 \frac{\alpha \pi\left(1-4 \alpha^{2}\right) \sin (\alpha \pi)+\left(1-12 \alpha^{2}\right) \cos (\alpha \pi)}{2}$ \\
\hline & $1-4 \alpha^{2}$ \\
\hline \multirow{2}{*}{ fexp } & {$[\ln (2)]^{3}+2 \cos (x)\left\{x^{4}+x^{2}[\ln (2)-3] \ln (2)-[\ln (2)]^{3}\right\}+x^{2} \ln (8)-2 x\left\{\left[[\ln (2)]^{3}+x^{2}[2+\ln (2)]\right]\right\} \sin (x)$} \\
\hline & $\left\{(\alpha \pi)^{2}+[\ln (2)]^{2}\right\}^{2}$ \\
\hline \multirow{2}{*}{ Poly $(b, c, d)$} & $\underline{120 d-12[2 b+3(c+d)] x^{2}-A \cos (x)+B \sin (x)}$ \\
\hline & $4 x^{4}$ \\
\hline \multirow{2}{*}{ Power } & $2+C\left\{\frac{2_{1}^{3+\beta} F_{2}\left[\{1\},\{(3+\beta) / 2,(4+\beta) / 2\},-(1 / 4) x^{2}\right]}{x^{2}}-2_{1}^{4+\beta} F_{2}\left[\{2\},\{(5+\beta) / 2,(6+\beta) / 2\},-(1 / 4) x^{2}\right]\right\}$ \\
\hline & $\sqrt{\pi} \Gamma[3+\beta]$ \\
\hline
\end{tabular}

formulae, which allows to obtain the first derivative of the time response as a closed-form expression. Table 2 reports the analytic expressions of the slope of the time response evaluated at $t / T=1$ for the pulses mentioned above, where $x=\alpha \pi$, and ${ }_{1} F_{2}[\{a\},\{b, c\}, y]$ is a hypergeometric function [8] given by

$$
{ }_{1} F_{2}[\{a\},\{b, c\}, y]=\sum_{k=0}^{\infty} \frac{(a)_{k}}{(b)_{k}(c)_{k}} \frac{y^{k}}{k !}
$$

with $(z)_{0}=1$ and $(z)_{k}=z(z+1)(z+2) \cdots(z+k-1)$.

For other pulses their impulse response is evaluated through a numerical inverse Fourier transform [3]. In this case one can use the slope interpretation to gain rough information about the derivative, which is the slope of the tangent line at the considered point.

In practice one often approximates the derivative by a difference quotient. After performing the numerical inverse Fourier transform the impulse response $h(t)$ is obtained as a set of discrete values $\left\{h_{k}\right\}$ at equally spaced time values given by the set $\left\{t_{k}\right\}$. If the sampling frequency was chosen high enough, say 100 samples per symbol interval, the points $\left\{h_{k}\right\}$ in the table are close enough together.

Given a table of values for the time response one can estimate the values of its derivative. So, the function does not change abruptly between them and the derivative is closely approximated by the difference quotient $s_{k}$ defined as

$$
s_{k}=\frac{h_{k+1}-h_{k-1}}{t_{k+1}-t_{k-1}},
$$

where $h_{k}$ is the sample of the impulse response at $t / T=1$, $h_{k+1}$ and $h_{k-1}$ are the samples adjacent to $h_{k}$.

The pulses reported in Table 2 that have a derivative expressed by a closed-form formula have been used for checking the accuracy of the calculations based on the difference quotient. Rel. (5) gave the best match.

\section{Second Derivative of the Time Response Evaluated at $t / T=1$}

We propose also to use the second derivative of the time response evaluated at $t / T=1$ as a figure of merit related to the size of the largest sidelobe. A bigger value of acceleration combined with a negative value of velocity, which is the case for the descending part of the largest sidelobe, will lead to a faster decrease of the time response. This results in increased performance when the time response is sampled with a timing error.

The second derivative of the impulse response was calculated for four pulses that give closed-form expressions of the time response and reported in Table 3 , where $x=\alpha \pi$, $A=120 d+12(c-2 b) x^{2}+(8+4 b+2 c+d) x^{4}, B=$ $2 x(24 c-12 d)+(8+8 b+2 c+d) x^{2}, C=2^{-(2+\beta)} \pi^{5 / 2} \alpha^{2} \Gamma[1+\beta]$, ${ }_{1} F_{2}[\{a\},\{b, c\}, y]$ is defined by rel. (4), and $\Gamma[t]$ is the Euler gamma function:

$$
\Gamma[t]=\int_{0}^{\infty} u^{(t-1)} e^{-u} d u .
$$

For other pulses that do not have time responses expressed by closed-form formulae the derivative of the slope $s_{k}$ at $t / T=1$ was obtained numerically. The value of the derivative of the slope $s_{k}$ at the time instant $t_{k}$, which can be envisaged as acceleration, was calculated as

$$
a_{k}=\frac{s_{k+1}-s_{k-1}}{t_{k+1}-t_{k-1}} \text {. }
$$

Comparing the values of the difference quotient $a_{k}$ with the values of the second derivative of the pulses determined with the closed-form formulae reported in Table 3 and evaluated at $t / T=1$, almost perfect match was found.

\section{Comparative Analysis of Several Improved Pulses}

In the sequel we have considered several improved pulses reported so far, such as RC, BTRC [1] also known as fexp [3], fsech [3], farcsech [3], Poly [5], acos [7], acos[acos] [7], $\operatorname{acos}[\operatorname{asech}]$ [7], $\operatorname{acos}[\log$ ] [7], asech[acos] [7], asech[asech] [7], asech[log] [7], asech [exp] [7], acos[exp] [7], Power [8], $\operatorname{acos}[\operatorname{asinh}]$ [6], acos[atan] [6], and sin[acosh] [6].

We have proceeded to rank the above mentioned pulses with respect to the error probability in descending order. We have correlated the results with the fractional energy defined as above or calculated by numerical integration.

Tables 4, 5, 6, and 7 report the values of error probability, fractional energy, and first and second derivatives of time response evaluated at $t / T=1$, for several cases of practical interest, namely, $\alpha=0.1, \alpha=0.25, \alpha=0.35$, and $\alpha=$ 0.5 , respectively, and three values of the normalized time offset, $0.05,0.1$, and 0.2 . For the sake of compactness we have denoted the normalized time offset $t / T$ as $\varepsilon$. 
TABLE 4: ISI error probabilities of several Nyquist pulses for $N=2^{9}$ interfering symbols, $T_{f}=40, M=61, \mathrm{SNR}=15 \mathrm{~dB}$, and $\alpha=0.1$.

\begin{tabular}{|c|c|c|c|c|c|c|}
\hline $\begin{array}{l}\text { Pulse } \\
\alpha=0.1\end{array}$ & $\begin{array}{c}P_{e} \\
\varepsilon=0.05\end{array}$ & $\begin{array}{c}P_{e} \\
\varepsilon=0.1\end{array}$ & $\begin{array}{c}P_{e} \\
\varepsilon=0.2\end{array}$ & $E_{s}$ & $\begin{array}{c}s_{k} \\
t / T=1\end{array}$ & $\begin{array}{c}a_{k} \\
t / T=1\end{array}$ \\
\hline $\mathrm{rcos}$ & $1.4130 e-7$ & $9.2400 e-6$ & $3.3488 e-3$ & 0.0182 & -0.9907 & 2.0185 \\
\hline fsech & $1.3583 e-7$ & $8.4906 e-6$ & $3.1092 e-3$ & 0.0205 & -0.9888 & 2.0222 \\
\hline $\operatorname{asech}[\log ]$ & $1.2292 e-7$ & $6.8473 e-6$ & $2.5495 e-3$ & 0.0245 & -0.9828 & 2.0340 \\
\hline fexp & $1.1926 e-7$ & $6.4133 e-6$ & $2.3933 e-3$ & 0.0279 & -0.9808 & 2.0381 \\
\hline farcsech & $1.1484 e-7$ & $5.9066 e-6$ & $2.2068 e-3$ & 0.0295 & -0.9779 & 2.0436 \\
\hline $\operatorname{acos}[\log ]$ & $1.1407 e-7$ & $5.8221 e-6$ & $2.1750 e-3$ & 0.0300 & -0.9774 & 2.0447 \\
\hline $\operatorname{acos}[\operatorname{atan}]$ & $1.1381 e-7$ & $5.7935 e-6$ & $2.1642 e-3$ & 0.0303 & -0.9772 & 2.0451 \\
\hline $\sin [\mathrm{acosh}]$ & $1.1360 e-7$ & $5.7710 e-6$ & $2.1557 e-3$ & 0.0305 & -0.9770 & 2.0454 \\
\hline $\operatorname{acos}[\operatorname{asinh}]$ & $1.1183 e-7$ & $5.5799 e-6$ & $2.0832 e-3$ & 0.0318 & -0.9755 & 2.0483 \\
\hline $\operatorname{acos}[\exp ]$ & $1.1126 e-7$ & $5.5190 e-6$ & $2.0599 e-3$ & 0.0323 & -0.9750 & 2.0493 \\
\hline $\operatorname{acos}[\operatorname{asech}]$ & $1.1083 e-7$ & $5.4740 e-6$ & $2.0427 e-3$ & 0.0325 & -0.9746 & 2.0500 \\
\hline $\operatorname{acos}$ & $1.1074 e-7$ & $5.4647 e-6$ & $2.0391 e-3$ & 0.0327 & -0.9745 & 2.0503 \\
\hline $\operatorname{acos}[\mathrm{acos}]$ & $1.0917 e-7$ & $5.3032 e-6$ & $1.9767 e-3$ & 0.0341 & -0.9730 & 2.0534 \\
\hline asech[exp] & $1.0879 e-7$ & $5.2647 e-6$ & $1.9617 e-3$ & 0.0345 & -0.9725 & 2.0542 \\
\hline asech[asech] & $1.0806 e-7$ & $5.1906 e-6$ & $1.9329 e-3$ & 0.0351 & -0.9718 & 2.0558 \\
\hline $\operatorname{asech}[\operatorname{acos}]$ & $1.0803 e-7$ & $5.1883 e-6$ & $1.9320 e-3$ & 0.0352 & -0.9717 & 2.0559 \\
\hline Poly $(60,-152,130)$ & $1.0355 e-7$ & $4.8248 e-6$ & $1.7843 e-3$ & 0.0437 & -0.9608 & 2.0773 \\
\hline Power $(\beta=0.07)$ & $1.0073 e-7$ & $4.5696 e-6$ & $1.6840 e-3$ & 0.0467 & -0.9558 & 2.0869 \\
\hline
\end{tabular}

TABLE 5: ISI error probabilities of several Nyquist pulses for $N=2^{9}$ interfering symbols, $T_{f}=40, M=61, \mathrm{SNR}=15 \mathrm{~dB}$, and $\alpha=0.25$.

\begin{tabular}{|c|c|c|c|c|c|c|}
\hline $\begin{array}{l}\text { Pulse } \\
\alpha=0.25\end{array}$ & $\begin{array}{c}P_{e} \\
\varepsilon=0.05\end{array}$ & $\begin{array}{c}P_{e} \\
\varepsilon=0.1 \\
\end{array}$ & $\begin{array}{c}P_{e} \\
\varepsilon=0.2 \\
\end{array}$ & $E_{s}$ & $\begin{array}{c}s_{k} \\
t / T=1\end{array}$ & $\begin{array}{c}a_{k} \\
t / T=1\end{array}$ \\
\hline $\operatorname{rcos}$ & $8.2189 e-8$ & $2.8184 e-6$ & $9.7462 e-4$ & 0.0454 & -0.9428 & 2.1095 \\
\hline fsech & $7.5579 e-8$ & $2.3337 e-6$ & $7.7201 e-4$ & 0.0512 & -0.9314 & 2.1310 \\
\hline asech[log] & $6.1687 e-8$ & $1.4808 e-6$ & $4.2640 e-4$ & 0.0613 & -0.8956 & 2.1938 \\
\hline fexp & $5.8117 e-8$ & $1.2980 e-6$ & $3.5678 e-4$ & 0.0697 & -0.8830 & 2.2187 \\
\hline farcsech & $5.3996 e-8$ & $1.1011 e-6$ & $2.8405 e-4$ & 0.0738 & -0.8664 & 2.2470 \\
\hline $\operatorname{acos}[\log ]$ & $5.3333 e-8$ & $1.0726 e-6$ & $2.7420 e-4$ & 0.0751 & -0.8630 & 2.2531 \\
\hline $\operatorname{acos}[\operatorname{atan}]$ & $5.3114 e-8$ & $1.0636 e-6$ & $2.7117 e-4$ & 0.0757 & -0.8617 & 2.2555 \\
\hline $\sin [\operatorname{acosh}]$ & $5.2941 e-8$ & $1.0565 e-6$ & $2.6878 e-4$ & 0.0762 & -0.8608 & 2.2573 \\
\hline $\operatorname{acos}[\operatorname{asinh}]$ & $5.1480 e-8$ & $9.9816 e-7$ & $2.4946 e-4$ & 0.0795 & -0.8520 & 2.2731 \\
\hline $\operatorname{acos}[\exp ]$ & $5.1036 e-8$ & $9.8109 e-7$ & $2.4406 e-4$ & 0.0807 & -0.8489 & 2.2787 \\
\hline $\operatorname{acos}$ [asech] & $5.0695 e-8$ & $9.6775 e-7$ & $2.3971 e-4$ & 0.0813 & -0.8467 & 2.2826 \\
\hline $\operatorname{acos}$ & $5.0636 e-8$ & $9.6618 e-7$ & $2.3940 e-4$ & 0.0817 & -0.8460 & 2.2838 \\
\hline $\operatorname{acos}[\mathrm{acos}]$ & $4.9480 e-8$ & $9.2585 e-7$ & $2.2747 e-4$ & 0.0852 & -0.8366 & 2.3008 \\
\hline $\operatorname{asech}[\exp ]$ & $4.9219 e-8$ & $9.1771 e-7$ & $2.2534 e-4$ & 0.0862 & -0.8340 & 2.3054 \\
\hline asech[asech] & $4.8700 e-8$ & $9.0060 e-7$ & $2.2053 e-4$ & 0.0877 & -0.8293 & 2.3138 \\
\hline $\operatorname{asech}[\operatorname{acos}]$ & $4.8697 e-8$ & $9.0126 e-7$ & $2.2096 \mathrm{e}-4$ & 0.0880 & -0.8289 & 2.3145 \\
\hline Poly $(39,-99,85)$ & $4.7582 e-8$ & $8.8156 e-7$ & $2.2060 e-4$ & 0.0937 & -0.8104 & 2.3468 \\
\hline Power $(\beta=0.29)$ & $4.6192 e-8$ & $8.2832 e-7$ & $2.0300 e-4$ & 0.0969 & -0.8002 & 2.3645 \\
\hline
\end{tabular}

Special attention must be paid to Poly and Power pulses, as they do not have a fixed form for a given value of $\alpha$, as the other pulses, but depend on one or three parameters, respectively. For the sake of compactness one usually adopted a set of truncated values determined for $\varepsilon=0.1$ and rounded to the next integer, which may lead to differences in performance.

The pulses have been placed in ascending order of performance with respect to error probability. We have found that for small values of the normalized time offset $\varepsilon=0.05$, 
TABLE 6: ISI error probabilities of several Nyquist pulses for $N=2^{9}$ interfering symbols, $T_{f}=40, M=61, \mathrm{SNR}=15 \mathrm{~dB}$, and $\alpha=0.35$.

\begin{tabular}{|c|c|c|c|c|c|c|}
\hline $\begin{array}{l}\text { Pulse } \\
\alpha=0.35\end{array}$ & $\begin{array}{c}P_{e} \\
\varepsilon=0.05\end{array}$ & $\begin{array}{c}P_{e} \\
\varepsilon=0.1\end{array}$ & $\begin{array}{c}P_{e} \\
\varepsilon=0.2\end{array}$ & $E_{s}$ & $\begin{array}{c}s_{k} \\
t / T=1\end{array}$ & $\begin{array}{c}a_{k} \\
t / T=1\end{array}$ \\
\hline $\cos$ & $5.9997 e-8$ & $1.3896 e-6$ & $3.9084 e-4$ & 0.0636 & -0.8902 & 2.2013 \\
\hline fsech & $5.4002 e-8$ & $1.0944 e-6$ & $2.8000 e-4$ & 0.0717 & -0.8684 & 2.2397 \\
\hline $\operatorname{asech}[\log ]$ & $4.2145 e-8$ & $6.2866 e-7$ & $1.2567 e-4$ & 0.0858 & -0.8023 & 2.3393 \\
\hline fexp & $3.9253 e-8$ & $5.4021 e-7$ & $1.0129 e-4$ & 0.0975 & -0.7777 & 2.3876 \\
\hline farcsech & $3.5970 e-8$ & $4.4580 e-7$ & $7.6203 e-5$ & 0.1033 & -0.7475 & 2.4301 \\
\hline $\operatorname{acos}[\log ]$ & $3.5470 e-8$ & $4.3365 e-7$ & $7.3486 e-5$ & 0.1051 & -0.7411 & 2.4403 \\
\hline $\operatorname{acos}[\operatorname{atan}]$ & $3.5310 e-8$ & $4.3008 e-7$ & $7.2778 e-5$ & 0.1060 & -0.7387 & 2.4445 \\
\hline $\sin [\operatorname{acosh}]$ & $3.5182 e-8$ & $4.2722 e-7$ & $7.2196 e-5$ & 0.1066 & -0.7369 & 2.4476 \\
\hline $\operatorname{acos}[\operatorname{asinh}]$ & $3.4124 e-8$ & $4.041 e-7$ & $6.7653 e-5$ & 0.1113 & -0.7205 & 2.4740 \\
\hline $\operatorname{acos}[\exp ]$ & $3.3806 e-8$ & $3.9786 e-7$ & $6.6617 e-5$ & 0.1123 & -0.7147 & 2.4834 \\
\hline $\operatorname{acos}[\operatorname{asech}]$ & $3.3558 e-8$ & $3.9255 e-7$ & $6.5582 e-5$ & 0.1139 & -0.7106 & 2.4894 \\
\hline $\operatorname{acos}$ & $3.3527 e-8$ & $3.9249 e-7$ & $6.5764 e-5$ & 0.1144 & -0.7094 & 2.4919 \\
\hline Poly $(31,-80,69)$ & $3.2897 e-8$ & $3.8388 e-7$ & $6.5629 e-5$ & 0.1174 & -0.6934 & 2.5132 \\
\hline Poly $(53,-134,113)$ & $3.2050 e-8$ & $4.1318 e-7$ & $8.7720 e-5$ & 0.1364 & -0.6129 & 2.6351 \\
\hline Poly $(36,-93,80)$ & $3.2461 e-8$ & $3.7915 e-7$ & $6.6140 e-5$ & 0.1203 & -0.6801 & 2.5326 \\
\hline Poly $(30.59,-78.27,67.2)$ & $3.2754 e-8$ & $3.8186 e-7$ & $6.5544 e-5$ & 0.1185 & -0.6896 & 2.5196 \\
\hline $\operatorname{acos}[\operatorname{acos}]$ & $3.2753 e-8$ & $3.7964 e-7$ & $6.4348 e-5$ & 0.1193 & -0.6918 & 2.5199 \\
\hline $\operatorname{asech}[\exp ]$ & $3.2591 e-8$ & $3.7775 e-7$ & $6.4445 e-5$ & 0.1207 & -0.6870 & 2.5277 \\
\hline $\operatorname{asech}[\operatorname{acos}]$ & $3.2264 e-8$ & $3.7363 e-7$ & $6.4494 \mathrm{e}-5$ & 0.1232 & -0.6774 & 2.5427 \\
\hline asech[asech] & $3.2255 e-8$ & $3.7275 e-7$ & $6.4110 e-5$ & 0.1229 & -0.6782 & 2.5412 \\
\hline Power $(\beta=0.4)$ & $3.1748 e-8$ & $3.6256 e-7$ & $6.2244 e-5$ & 0.1250 & -0.6683 & 2.5551 \\
\hline Power $(\beta=0.23)$ & $3.0524 e-8$ & $3.6990 e-7$ & $7.4044 e-5$ & 0.1423 & -0.5968 & 2.6616 \\
\hline Power $(\beta=0.32)$ & $3.0955 e-8$ & $3.5466 e-7$ & $6.4326 e-5$ & 0.1326 & -0.6373 & 2.6015 \\
\hline Power $(\beta=0.39)$ & $3.1629 e-8$ & $3.6109 e-7$ & $6.1940 e-5$ & 0.1259 & -0.6646 & 2.5606 \\
\hline
\end{tabular}

TABLE 7: ISI error probabilities of several Nyquist pulses for $N=2^{9}$ interfering symbols, $T_{f}=40, M=61, \mathrm{SNR}=15 \mathrm{~dB}$, and $\alpha=0.5$.

\begin{tabular}{|c|c|c|c|c|c|c|}
\hline $\begin{array}{l}\text { Pulse } \\
\alpha=0.5\end{array}$ & $\begin{array}{c}P_{e} \\
\varepsilon=0.05\end{array}$ & $\begin{array}{c}P_{e} \\
\varepsilon=0.1\end{array}$ & $\begin{array}{c}P_{e} \\
\varepsilon=0.2\end{array}$ & $E_{s}$ & $\begin{array}{c}s_{k} \\
t / T=1\end{array}$ & $\begin{array}{c}a_{k} \\
t / T=1\end{array}$ \\
\hline $\mathrm{rcos}$ & $3.9723 e-8$ & $5.4890 e-7$ & $1.0217 e-4$ & 0.0908 & -0.7854 & 2.3562 \\
\hline fsech & $3.4949 e-8$ & $4.1186 e-7$ & $6.6009 e-5$ & 0.1024 & -0.7436 & 2.4194 \\
\hline asech[log] & $2.6157 e-8$ & $2.1763 e-7$ & $2.5364 e-5$ & 0.1226 & -0.6253 & 2.5316 \\
\hline fexp & $2.4134 e-8$ & $1.8580 e-7$ & $2.0878 e-5$ & 0.1393 & -0.5757 & 2.6256 \\
\hline farcsech & $2.1875 e-8$ & $1.4916 e-7$ & $1.5344 e-5$ & 0.1476 & -0.5231 & 2.6629 \\
\hline $\operatorname{acos}[\log ]$ & $2.1559 e-8$ & $1.4514 e-7$ & $1.4987 e-5$ & 0.1502 & -0.5114 & 2.6768 \\
\hline $\operatorname{acos}[\operatorname{atan}]$ & $2.1462 e-8$ & $1.4410 e-7$ & $1.4953 e-5$ & 0.1515 & -0.5068 & 2.6836 \\
\hline $\sin [\operatorname{acosh}]$ & $2.1386 e-8$ & $1.4323 e-7$ & $1.4911 e-5$ & 0.1523 & -0.5033 & 2.6882 \\
\hline $\operatorname{acos}[\operatorname{asinh}]$ & $2.0758 e-8$ & $1.3617 e-7$ & $1.4609 e-5$ & 0.1590 & -0.4731 & 2.7239 \\
\hline $\operatorname{acos}[\exp ]$ & $2.0583 e-8$ & $1.3446 e-7$ & $1.4657 \mathrm{e}-5$ & 0.1614 & -0.4624 & 2.7368 \\
\hline Poly $(25,-64,55)$ & $2.0574 e-8$ & $1.3539 e-7$ & $1.5197 \mathrm{e}-5$ & 0.1615 & -0.4575 & 2.7316 \\
\hline $\operatorname{acos}[\operatorname{asech}]$ & $2.0438 e-8$ & $1.3273 e-7$ & $1.4563 e-5$ & 0.1627 & -0.4552 & 2.7434 \\
\hline $\operatorname{acos}$ & $2.0431 e-8$ & $1.3300 \mathrm{e}-7$ & $1.4717 \mathrm{e}-5$ & 0.1635 & -0.4527 & 2.7479 \\
\hline $\operatorname{acos}[\mathrm{acos}]$ & $2.0054 e-8$ & $1.3014 e-7$ & $1.5328 \mathrm{e}-5$ & 0.1705 & -0.4205 & 2.7850 \\
\hline $\operatorname{asech}[\exp ]$ & $1.9992 e-8$ & $1.3005 e-7$ & $1.5658 \mathrm{e}-5$ & 0.1724 & -0.4116 & 2.7958 \\
\hline $\operatorname{asech}[\operatorname{acos}]$ & $1.9865 e-8$ & $1.2958 e-7$ & $1.6248 \mathrm{e}-5$ & 0.1761 & -0.3942 & 2.8119 \\
\hline asech[asech] & $1.9845 e-8$ & $1.2902 e-7$ & $1.6057 \mathrm{e}-5$ & 0.1756 & -0.3957 & 2.8148 \\
\hline Power $(\beta=0.37)$ & $1.9451 e-8$ & $1.2504 e-7$ & $1.6619 e-5$ & 0.1825 & -0.3586 & 2.8428 \\
\hline Power $(\beta=0.32)$ & $1.9356 e-8$ & $1.2645 e-7$ & $1.8540 \mathrm{e}-5$ & 0.1894 & -0.3231 & 2.8759 \\
\hline Power $(\beta=0.37)$ & $1.9451 e-8$ & $1.2469 e-7$ & $1.6619 e-5$ & 0.1825 & -0.3586 & 2.8428 \\
\hline
\end{tabular}


TABLE 8: Comparative performance of several Nyquist pulses for $\alpha=0.25, \varepsilon=0.1$, and $\varepsilon=0.2$.

\begin{tabular}{|c|c|c|c|c|c|c|c|c|c|c|c|}
\hline$\alpha=0.25$ & Pulse & $P_{e}$ & $E_{s}$ & $h(\varepsilon)$ & $d_{1}^{\varepsilon}$ & $d_{2}^{\varepsilon}$ & $d_{3}^{\varepsilon}$ & $d_{4}^{\varepsilon}$ & $d_{5}^{\varepsilon}$ & $d_{7}^{\varepsilon}$ & $d_{10}^{\varepsilon}$ \\
\hline \multirow{2}{*}{$\varepsilon=0.1$} & asech[asech] & $9.0060 \mathrm{e}-7$ & 0.0877 & 0.9819 & 0.0711 & 0.0873 & 0.0920 & 0.1034 & 0.1133 & 0.1186 & 0.1287 \\
\hline & asech[acos] & $9.0126 e-7$ & 0.0880 & 0.9819 & 0.0711 & 0.0871 & 0.0920 & 0.1035 & 0.1135 & 0.1189 & 0.1289 \\
\hline \multirow{2}{*}{$\varepsilon=0.2$} & asech[asech] & $2.2053 e-4$ & 0.0877 & 0.9289 & 0.1182 & 0.1432 & 0.1545 & 0.1764 & 0.1944 & 0.2043 & 0.2231 \\
\hline & asech[acos] & $2.2096 e-4$ & 0.0880 & 0.9288 & 0.1182 & 0.1430 & 0.1545 & 0.1766 & 0.1949 & 0.2049 & 0.2235 \\
\hline
\end{tabular}

$\varepsilon=0.1$, and $\varepsilon=0.2$, the pulses reported in ascending order of performance with respect to error probability are also placed in ascending order of fractional energy $E_{s}$, descending order of the absolute value of the slope $s_{k}$ of the impulse response evaluated at $t / T=1$, and ascending order of the second derivative of the impulse response $a_{k}$ evaluated at $t / T=1$.

However, for $\alpha=0.25$, and $\varepsilon \geq 0.1$ there are two exceptions in Table 5 marked in bold characters, involving the asech[asech] and asech[acos] pulses with close values of fractional energy $E_{s}$ of 0.0877 , and 0.0880 , respectively. The values of error probability evaluated for $\varepsilon=0.1$ and $\varepsilon=0.2$ do not obey the ranking order given by the fractional energy $E_{s}$, the slope value $s_{k}$ at $t / T=1$, and acceleration $a_{k}$ at $t / T=1$. Despite its higher values of $E_{s}, s_{k}$, and $a_{k}$, the asech[acos] pulse is slightly outperformed by the asech[asech] pulse for timing offsets of 0.1 and 0.2 .

The bold characters mark the situation that despite better values of $E_{s}, s_{k}$, and $a_{k}$, the value of error probability of asech[acos] pulse for $\varepsilon \geq 0.1$ is worse with respect to the asech[asech] pulse, while for $\varepsilon=0.05$ this correlation between error probability and the $E_{s}, s_{k}$, and $a_{k}$ values holds. Although the difference in error probability for $\varepsilon=0.2$ is quite small, around $1.4 \%$, this deserves further analysis.

\section{Further Analysis}

It is worth mentioning that the acos[acos], asech[exp], asech[acos], and asech[asech] pulses have almost identical frequency characteristics, which results in very close values of the error probability, the difference in error probability values being under $0.05 \%$ for small values of the normalized time offset.

The difference in the values of the main lobe sample $h(\varepsilon)$ is very small, so to explain the difference in performance we have introduced the limited ISI distortion for a time offset $\varepsilon$, produced by $k$ interfering pulses denoted as $d_{k}^{\varepsilon}$, and defined as

$$
d_{k}^{\varepsilon}=\sum_{i=1}^{k}|h(i+\varepsilon)| .
$$

The absolute value of the sample of the $n$th lobe for a specified value of $\varepsilon$ can be found also as

$$
|h(n+\varepsilon)|=d_{n}^{\varepsilon}-d_{n-1}^{\varepsilon} .
$$

For instance $|h(4.1)|$ for asech[asech] pulse is found as $|h(4.1)|=d_{4}^{0.1}-d_{3}^{0.1}=0.1034-0.0920=0.0114$, while for asech[acos] pulse $|h(4.1)|=0.0115$.

To understand what determines the difference in performance for pulses with almost the same value of the error probability and fractional energy, we have calculated the values of the main lobe sample $h(\varepsilon)$ and of $d_{k}^{\varepsilon}$, with $\varepsilon=0.1$ and $\varepsilon=0.2$, for a pair of pulses with almost equal performance in terms of error probability, namely, asech[asech] and asech[acos] that do not obey the proposed ranking criteria and also for the Poly pulse that shows a greater value of error probability, as expected based on fractional energy value for $\varepsilon=0.2$. This is shown in Table 8 that presents a comparative analysis of asech[asech], and asech[acos] and Poly pulses for the excess bandwidth value $\alpha=0.25$. The entries in bold characters specify the advantages of a pulse with respect to the other one in a set of pulses with almost the same value of the error probability and fractional energy.

Sampling the Nyquist pulse at the receiver's site with a time offset $\varepsilon$, that is, sampling off-peak incurs a penalty, which can be seen in the value of $h(\varepsilon)$. For $\varepsilon=0.2$ the samples are taken far from the center of the eye and the value of $h(\varepsilon)$ is decreased.

There are two factors that contribute to the error mechanism. One is the decreased value of the main sample $h(\varepsilon)$ and the other one is the residual effect of all other transmitted bits, known as ISI. The improved pulses have an asymptotic decay rate of $t^{-2}$ when sampled with a timing offset. So, the higher order sidelobes are less important than the low order sidelobes in determining the error performance.

An analysis of the pulses reported in Table 8 reveals that asech[asech] and asech[acos] pulses have almost equal performance. As the sample of the main lobe $h(\varepsilon)$ is almost the same, the small difference in performance may be attributed to the difference in the absolute values of the sample of the low order sidelobes, say 3 th to 10 th.

For $\varepsilon=0.2$ the asech[asech] pulse has an advantage also due to the slightly higher value of $h(0.2)$.

Although the asech[acos] pulse has a smaller value of $d_{2}^{0.2}$ than asech[asech] pulse and the same value of $d_{1}^{0.2}$, its performance is worse due to the higher values of $d_{k}^{0.2}, k>3$. A higher value of $d_{k}^{\varepsilon}$ evidences that, for the specified time offset $\varepsilon$, the sum of absolute values of the samples of the sidelobes up to $k$ th sidelobe is larger and produces more ISI that results in increased error probability value.

The improved behavior is explained in [2] by examination of the inner sidelobes of the pulses. Improved performance is obtained if the magnitudes of the two largest sidelobes of the pulse under consideration are smaller as compared with the magnitudes of the two largest sidelobes of the raisedcosine pulse [1]. Also the maximum distortion is less for the improved pulses than the raised-cosine pulse [2].

When dealing with improved pulses of almost equal performance this is not enough and one must also consider 
TABLE 9: Comparative performance of several Nyquist pulses for $\alpha=0.35, \varepsilon=0.1$, and $\varepsilon=0.2$.

\begin{tabular}{cccccccccccc}
\hline$\alpha=0.35$ & Pulse & $P_{e}$ & $E_{s}$ & $h(\varepsilon)$ & $d_{1}^{\varepsilon}$ & $d_{2}^{\varepsilon}$ & $d_{3}^{\varepsilon}$ & $d_{4}^{\varepsilon}$ & $d_{5}^{\varepsilon}$ & $d_{7}^{\varepsilon}$ & $d_{10}^{\varepsilon}$ \\
\hline \multirow{5}{*}{$\varepsilon=0.1$} & asech[asech] & $\mathbf{3 . 7 2 7 5 e}-\mathbf{7}$ & 0.1229 & 0.9802 & 0.0552 & 0.0588 & $\mathbf{0 . 0 7 5 0}$ & $\mathbf{0 . 0 8 4 4}$ & $\mathbf{0 . 0 8 5 7}$ & $\mathbf{0 . 0 9 5 2}$ & $\mathbf{0 . 1 0 1 9}$ \\
& asech[acos] & $3.7363 e-7$ & $\mathbf{0 . 1 2 3 2}$ & 0.9802 & $\mathbf{0 . 0 5 5 1}$ & $\mathbf{0 . 0 5 5 8}$ & 0.0753 & 0.0848 & 0.0860 & 0.0954 & 0.1021 \\
& acos[asech] & $3.9255 e-7$ & 0.1139 & 0.9795 & 0.0484 & $\mathbf{0 . 0 5 9 3}$ & $\mathbf{0 . 0 7 7 7}$ & $\mathbf{0 . 0 8 3 3}$ & $\mathbf{0 . 0 9 0 3}$ & $\mathbf{0 . 1 0 0 2}$ & $\mathbf{0 . 1 1 0 6}$ \\
& acos & $\mathbf{3 . 9 2 4 9 e}-7$ & $\mathbf{0 . 1 1 4 4}$ & 0.9794 & $\mathbf{0 . 0 4 8 1}$ & 0.0594 & 0.0782 & 0.0840 & 0.0909 & 0.1008 & 0.1113 \\
\hline \multirow{6}{*}{$\varepsilon=0.2$} & acos[acos] & $\mathbf{6 . 4 3 4 8 e}-\mathbf{5}$ & 0.1193 & $\mathbf{0 . 9 1 8 8}$ & 0.0682 & $\mathbf{0 . 0 9 8 7}$ & $\mathbf{0 . 1 3 5 9}$ & $\mathbf{0 . 1 4 4 1}$ & $\mathbf{0 . 1 5 9 9}$ & $\mathbf{0 . 1 7 7 3}$ & $\mathbf{0 . 2 0 0 0}$ \\
& asech[exp] & $6.4445 e-5$ & $\mathbf{0 . 1 2 0 7}$ & 0.9186 & $\mathbf{0 . 0 6 7 0}$ & 0.0990 & 0.1370 & 0.1454 & 0.1615 & 0.1792 & 0.2023 \\
& asech[asech] & $\mathbf{6 . 4 1 1 0 e}-\mathbf{5}$ & 0.1229 & 0.9225 & 0.0862 & $\mathbf{0 . 0 9 8 1}$ & $\mathbf{0 . 1 2 8 8}$ & $\mathbf{0 . 1 4 4 9}$ & $\mathbf{0 . 1 4 8 5}$ & $\mathbf{0 . 1 6 5 9}$ & $\mathbf{0 . 1 7 8 7}$ \\
& asech[acos] & $\mathbf{6 . 4 4 9 4 e - 5}$ & $\mathbf{0 . 1 2 3 2}$ & 0.9225 & 0.0860 & 0.0982 & 0.1293 & 0.1353 & 0.1491 & 0.1664 & 0.1791 \\
& acos[asech] & $\mathbf{6 . 5 5 8 2 e}-\mathbf{5}$ & 0.1139 & $\mathbf{0 . 9 1 9 7}$ & 0.0726 & $\mathbf{0 . 0 9 7 8}$ & $\mathbf{0 . 1 3 1 5}$ & $\mathbf{0 . 1 3 9 2}$ & $\mathbf{0 . 1 5 3 7}$ & $\mathbf{0 . 1 7 0 1}$ & $\mathbf{0 . 1 9 1 0}$ \\
& acos & $6.5764 e-5$ & 0.1144 & 0.9196 & 0.0720 & 0.0980 & 0.1324 & 0.1404 & 0.1549 & 0.1713 & 0.1923 \\
\hline
\end{tabular}

TABLE 10: Comparative performance of several Nyquist pulses for $\alpha=0.5, \varepsilon=0.1$, and $\varepsilon=0.2$.

\begin{tabular}{|c|c|c|c|c|c|c|c|c|c|c|c|}
\hline$\alpha=0.5$ & Pulse & $P_{e}$ & $E_{s}$ & $h(\varepsilon)$ & $d_{1}^{\varepsilon}$ & $d_{2}^{\varepsilon}$ & $d_{3}^{\varepsilon}$ & $d_{4}^{\varepsilon}$ & $d_{5}^{\varepsilon}$ & $d_{7}^{\varepsilon}$ & $d_{10}^{\varepsilon}$ \\
\hline \multirow{2}{*}{$\varepsilon=0.1$} & acos[asech] & $1.3273 e-7$ & 0.1627 & 0.9774 & 0.0324 & 0.0500 & 0.0571 & 0.0636 & 0.0670 & 0.0727 & 0.0782 \\
\hline & $\mathrm{acos}$ & $1.3300 e-7$ & 0.1635 & 0.9773 & 0.0321 & 0.0501 & 0.0576 & 0.0640 & 0.0673 & 0.0730 & 0.0784 \\
\hline \multirow{8}{*}{$\varepsilon=0.2$} & $\operatorname{acos}[\operatorname{asinh}]$ & $1.4609 e-5$ & 0.1590 & 0.9126 & 0.0455 & 0.0772 & 0.0873 & 0.0993 & 0.1039 & 0.1136 & 0.1232 \\
\hline & $\operatorname{acos}[\exp ]$ & $1.4657 e-5$ & 0.1614 & 0.9121 & 0.0433 & 0.0769 & 0.0874 & 0.0997 & 0.1045 & 0.1145 & 0.1244 \\
\hline & $\operatorname{acos}[$ asech] & $1.4563 e-5$ & 0.1627 & 0.9118 & 0.0419 & 0.0763 & 0.0862 & 0.0994 & 0.1042 & 0.1145 & 0.1249 \\
\hline & $\mathrm{acos}$ & $1.4717 e-5$ & 0.1635 & 0.9117 & 0.0413 & 0.0765 & 0.0871 & 0.0999 & 0.1048 & 0.1151 & 0.1254 \\
\hline & $\operatorname{acos}[\mathrm{acos}]$ & $1.5328 \mathrm{e}-5$ & 0.1705 & 0.9102 & 0.0348 & 0.0752 & 0.0864 & 0.1008 & 0.1059 & 0.1174 & 0.1289 \\
\hline & $\operatorname{asech}[\exp ]$ & $1.5658 e-5$ & 0.1724 & 0.9098 & 0.0329 & 0.0749 & 0.0865 & 0.1012 & 0.1064 & 0.1182 & 0.1299 \\
\hline & asech[asech] & $1.6057 \mathrm{e}-5$ & 0.1756 & 0.9091 & 0.0297 & 0.0738 & 0.0848 & 0.1008 & 0.1061 & 0.1187 & 0.1312 \\
\hline & asech $[\mathrm{acos}]$ & $1.6248 e-5$ & 0.1761 & 0.9090 & 0.0294 & 0.0740 & 0.0854 & 0.1013 & 0.1066 & 0.1191 & 0.1316 \\
\hline
\end{tabular}

the magnitudes of the samples of the low order sidelobes, up to the 10th side lobe.

For $\alpha=0.35$ several exceptions regarding the order marked in bold characters were evidenced, involving acos[acos], acos, asech[exp], asech[acos], and asech[asech] pulses.

In Table 9 we have considered the asech[asech], asech[acos], acos[asech], acos, acos[acos], and asech[exp] pulses for the cases when they do not obey the ranking rule based on fractional energy and values of the first and second derivative evaluated at $t / T=1$.

For $\alpha=0.35$ and $\varepsilon=0.1$, despite having a larger value of $E_{s}$, the asech[acos] pulse is slightly outperformed by the asech[asech] pulse. This can be attributed to the larger value of $d_{k}^{\varepsilon}, 3 \leq k \leq 10$ for asech[acos] pulse.

For small values of timing offset, $\varepsilon \leq 0.1$, the fractional energy criterion is prevalent. For higher values of timing offset and close values of the fractional energy one should also consider the limited ISI distortion $d_{k}^{\varepsilon}$ with $k$ up to 10 .

The values of error probability, fractional energy, slope $s_{k}$, and acceleration $a_{k}$ are tabulated in Table 7 for $\alpha=0.5$.

Table 10 presents a comparative analysis of several pairs of pulses with close values of fractional energy from Table 7 for the cases when they do not obey the proposed ranking criteria.

The advantage of a pulse over the other one in the same pair was marked in bold characters, that is, higher values of $E_{s}$ and main lobe sample on one hand and lower absolute values of the samples of sidelobes up to the $k$ th one on the other hand.

As inferred from Table 10, for close values of fractional energy, the pulse with a smaller value of the fractional energy outperforms the other pulse in the pair for higher values of the timing offset due to a decrease in the magnitude of the side lobes up to the $k$ th one, with $k$ ranging in the interval $[4,10]$ and a slight increase in the magnitude of the main lobe sample for some pulses.

\section{Power Sine Pulses}

In order to check that the fractional energy can be applied to the design of an improved Nyquist pulse, a novel family of pulses known as power sine (PS) was proposed. The PS pulse shows a frequency characteristic that is concave in the frequency range $[B(1-\alpha), B]$. So, an energy transfer is made from the lower frequency range $[B(1-\alpha), B]$ to the higher one $[B, B(1+\alpha)]$ in order of the odd symmetry of the Nyquist frequency characteristic. As a result, improved performance is expected as compared with the RC pulse $[7,9]$.

Its frequency spectrum is defined as

$$
\begin{aligned}
& S_{\mathrm{PS}}(f) \\
& = \begin{cases}1, & |f| \leq B(1-\alpha), \\
1-\frac{1}{2}\left(\sin \left[\pi \frac{f-B(1-\alpha)}{2 B \alpha}\right]\right)^{n}, & B(1-\alpha)<|f| \leq B, \\
\frac{1}{2}\left(\sin \left[\pi \frac{B(1+\alpha)-f}{2 B \alpha}\right]\right)^{n}, & B<|f| \leq B(1+\alpha), \\
0, & B(1+\alpha)<|f| .\end{cases}
\end{aligned}
$$




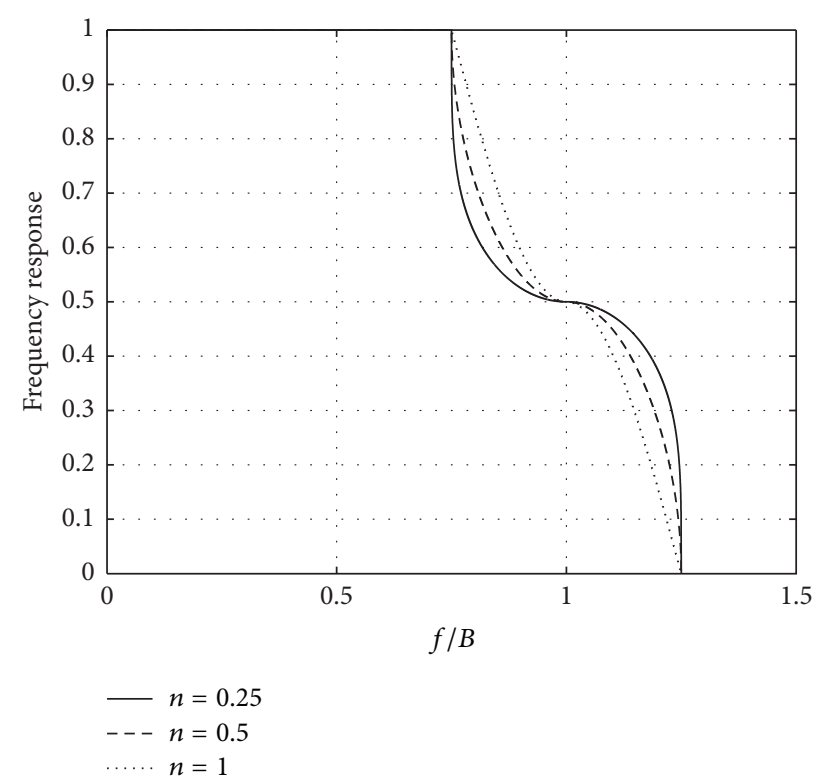

FIGURE 1: Frequency characteristics of the PS pulse for an excess bandwidth $\alpha=0.25$ and $n=0.25,0.5$, and 1 .

Figure 1 illustrates the frequency characteristic of PS pulse for $\alpha=0.25$ and $n=0.25,0.5$, and 1 .

The design of an improved Nyquist pulse with an impulse response that depends on several parameters and not given by a closed-form expression is a time-consuming process. The impulse response must be evaluated through a numerical inverse Fourier transform for a given set of parameters and then one calculates the error probability when the impulse response is sampled with a time offset $\varepsilon$. If $\varepsilon=0.05$ this implies the use of 20 samples per symbol interval.

Usually the average symbol error probabilities of binary antipodal signaling [12] in the presence of symbol timing error implies the use of 512 interfering symbols, so the impulse response is described by a vector with 10240 components. The process must be repeated many times using different values of parameters. Consider the PS pulse. The parameters involved in the calculation of the error probability of PS pulse are the excess bandwidth $\alpha$ and the exponent $n$.

In order to expedite the design process of a PS filter with good performance for a specified value of $\alpha$, one should determine the values of $n$ for which fractional energy is optimal.

In other cases, if $E_{s}$ cannot be obtained as a closedform expression, one should use numerical integration and calculate it for a set of values.

The fractional energy of PS pulse was obtained as

$$
E_{s}=\frac{\alpha}{2} \frac{\Gamma[(1+n) / 2]}{\sqrt{\pi} \Gamma[1+n / 2]} .
$$

In order to get improved performance, one should first obtain the impulse response using a numerical integration of the frequency characteristic for a set of values of $n$. This is a time-consuming operation followed by the calculation of the error probability and the comparison with the best pulses reported so far, for example, Poly [5] and Power [8].

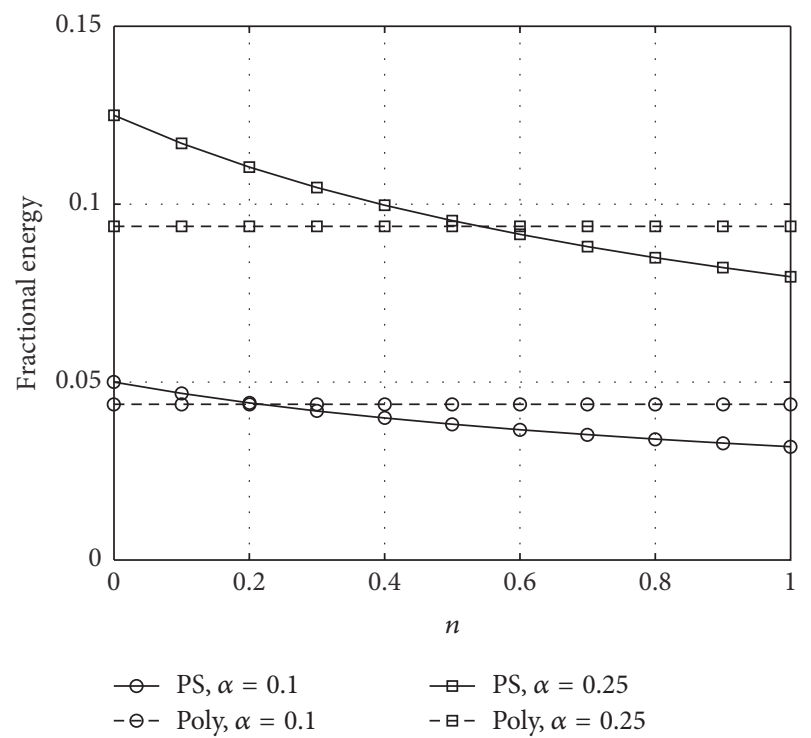

FIGURE 2: Variation of fractional energy of PS pulse and optimal Poly pulse for $\alpha=0.1$ and $\alpha=0.25$.

To speed up the design process one should reduce the set size of $n$ values.

Assume $\alpha=0.1$ and that we want to obtain a PS pulse that outperforms the Poly pulse. The fractional energy value of the optimal Poly pulse for $\alpha=0.1$ is 0.0437 .

Figure 2 illustrates the variation of $E_{s}$ with $n$ for the PS pulse for $\alpha=0.1$ and $\alpha=0.25$.

Assuming a 10\% error margin, we need to determine the error probability for values of $n$ around 0.25 and below it for $\alpha=0.1$. The results are presented in Table 11 .

One can see that the PS pulse outperforms the Poly pulse for $n \leq 0.3$ but it is outperformed by the Power pulse, despite its greater value of $E_{s}$. As the error probability values are very close to each other, it is obvious that there are other factors to be considered.

Assume $\alpha=0.25$. The Poly pulse has $E_{s}=0.0937$. The PS pulse will have a maximum of performance if its fractional energy $E_{s}$ is close to this value. This reaches 0.09375 for $n=$ 0.54 . Assuming a $10 \%$ error margin, we need to determine the error probability for values of $n$ around 0.6 and below it. The results are tabulated in Table 12.

For $\alpha=0.25, \varepsilon=0.05, n=0.2$, and $n=0.25$ the PS pulse outperforms both Poly and Power pulses.

\section{Conclusions}

To explain the improved performance of Nyquist pulses in the presence of timing errors three figures of merit based on fractional energy, first and second derivative of the time response, in short velocity and acceleration, evaluated at first zero crossing of the impulse response have been proposed and verified on pulses that have been reported in the corresponding literature and on a novel family of pulses. Small discrepancies appear for higher values of the excess bandwidth and symbol timing errors. All three figures of merit indicate the energy distribution into the sidelobes 
TABLE 11: ISI error probabilities of PS Nyquist pulses for $N=2^{9}$ interfering symbols, $T_{f}=40, M=61, \mathrm{SNR}=15 \mathrm{~dB}$, and $\alpha=0.1$.

\begin{tabular}{|c|c|c|c|c|}
\hline $\begin{array}{l}\text { Pulse } \\
\alpha=0.1\end{array}$ & $\begin{array}{c}P_{e} \\
\varepsilon=0.05\end{array}$ & $\begin{array}{c}P_{e} \\
\varepsilon=0.1\end{array}$ & $\begin{array}{c}P_{e} \\
\varepsilon=0.2\end{array}$ & $E_{s}$ \\
\hline PS $(n=0.05)$ & $1.0098 \mathrm{e}-7$ & $4.6239 e-6$ & $1.7038 e-3$ & 0.0483 \\
\hline PS $(n=0.1)$ & $1.0091 \mathrm{e}-7$ & $4.5877 e-7$ & $1.6911 e-3$ & 0.0468 \\
\hline PS $(n=0.2)$ & $1.0159 \mathrm{e}-7$ & $4.6133 e-6$ & $1.7031 e-3$ & 0.0442 \\
\hline PS $(n=0.25)$ & $1.0220 \mathrm{e}-7$ & $4.6579 e-6$ & $1.7213 e-3$ & 0.0430 \\
\hline PS $(n=0.3)$ & $1.0291 \mathrm{e}-7$ & $4.7162 e-6$ & $1.7448 e-3$ & 0.0419 \\
\hline PS $(n=0.35)$ & $1.0369 e-7$ & $4.7847 e-6$ & $1.7721 e-3$ & 0.0408 \\
\hline PS $(n=0.4)$ & $1.0452 e-7$ & $4.8607 e-6$ & $1.8023 e-3$ & 0.0399 \\
\hline Poly $(60,-152,130)$ & $1.0355 e-7$ & $4.8248 e-6$ & $1.7843 e-3$ & 0.0437 \\
\hline Power $(\beta=0.07)$ & $1.0073 e-7$ & $4.5696 e-6$ & $1.6840 e-3$ & 0.0467 \\
\hline
\end{tabular}

TABLE 12: ISI error probabilities of PS Nyquist pulses for $N=2^{9}$ interfering symbols, $T_{f}=40, M=61, \mathrm{SNR}=15 \mathrm{~dB}$, and $\alpha=0.25$.

\begin{tabular}{|c|c|c|c|c|}
\hline $\begin{array}{l}\text { Pulse } \\
\alpha=0.25\end{array}$ & $\begin{array}{c}P_{e} \\
\varepsilon=0.05\end{array}$ & $\begin{array}{c}P_{e} \\
\varepsilon=0.1\end{array}$ & $\begin{array}{c}P_{e} \\
\varepsilon=0.2\end{array}$ & $E_{s}$ \\
\hline PS $(n=0.1)$ & $4.6771 e-8$ & $9.7161 e-7$ & $2.8226 e-4$ & 0.1171 \\
\hline PS $(n=0.2)$ & $4.5937 \mathrm{e}-8$ & $8.8541 e-8$ & $2.3924 e-4$ & 0.1104 \\
\hline PS $(n=0.25)$ & $4.5938 \mathrm{e}-8$ & $8.6765 e-7$ & $2.2871 e-4$ & 0.1074 \\
\hline PS $(n=0.3)$ & $4.6119 e-8$ & $8.6054 e-7$ & $2.2271 e-4$ & 0.1047 \\
\hline PS $(n=0.35)$ & $4.6435 e-8$ & $8.6133 e-7$ & $2.2006 e-4$ & 0.1021 \\
\hline $\operatorname{PS}(n=0.4)$ & $4.6852 e-8$ & $8.6817 e-7$ & $2.1996 e-4$ & 0.0997 \\
\hline PS $(n=0.45)$ & $4.7346 e-8$ & $8.7972 e-7$ & $2.2187 e-4$ & 0.0975 \\
\hline PS $(n=0.5)$ & $4.7897 e-8$ & $8.9501 e-7$ & $2.2537 e-4$ & 0.0953 \\
\hline PS $(n=0.55)$ & $4.8494 e-8$ & $9.1334 e-7$ & $2.3018 e-4$ & 0.0934 \\
\hline Poly $(39,-99,85)$ & $4.7582 e-8$ & $8.8156 e-7$ & $2.2060 e-4$ & 0.0937 \\
\hline Power $(\beta=0.29)$ & $4.6192 e-8$ & $8.2832 e-7$ & $2.0300 e-4$ & 0.0969 \\
\hline
\end{tabular}

of the time response that leads to improved performance when the impulse response is sampled with a time offset and are related to the pulse performance. The mechanism of energy distribution based on fractional energy, velocity, and acceleration, evaluated at first zero crossing of the impulse response, works very well for small and moderate values of excess bandwidth and time offset. So, all these criteria can be used for quick performance assessment of improved Nyquist pulses. For small values of excess bandwidth, say $\alpha=0.1$ and $\alpha=0.25$, the proposed criteria based on fractional energy and values of the first and second derivative of the time response evaluated at $t / T=1$ are valid to rank the pulses based on error probability performance when sampled with usual values of the time offset, say $0.05,0.1$, and 0.2 . Caution must be exerted when investigating pulses with close values of fractional energy, as exceptions may occur. Also the criteria based on the values of the first and second derivative of the time response evaluated at $t / T=1$ (velocity and acceleration) must be used with caution taking into account that they are not independent. For instance, considering a pair of pulses with close values of fractional energy, velocity and acceleration, different results may be obtained taking into account that the decrease of the magnitude of the largest side lobe can be produced by either a bigger value of velocity combined with a smaller value of acceleration or vice versa.
Also, the proposed criteria for ranking the pulses should be used with caution when comparing pulses with different asymptotic decay rates at higher values of excess bandwidth $\alpha$. For larger values of excess bandwidth $\alpha$ and higher values of the time offset, say $t / T=0.2$, when the pulses have values of $E_{s}$ that are close to each other, the difference in error probability is no longer determined only by the magnitude of the two largest sidelobes [7], but also by the differences in the magnitude of the sidelobes up to the $k$ th one, with $3 \leq k \leq 10$. The quick assessment method proposed here was verified on a novel family of improved Nyquist pulses, denoted as power sine. This significantly restricted the value range of the design parameter $n$ to be searched in order to obtain improved performance. To explain the difference in pulse performance a figure of merit based on the limited ISI distortion was introduced. The mechanism of performance improvement was not completely deciphered so far, as for each improved pulse there is an optimal value of $E_{s}, s_{k}$, and $a_{k}$ that once surpassed may lead to poorer performance.

\section{Competing Interests}

The authors declare that there is no conflict of interests regarding the publication of this paper. 


\section{References}

[1] N. C. Beaulieu, C. C. Tan, and M. O. Damen, "A better than Nyquist pulse," IEEE Communications Letters, vol. 5, no. 9, pp. 367-368, 2001.

[2] N. C. Beaulieu and M. O. Damen, "Parametric construction of Nyquist-I pulses," IEEE Transactions on Communications, vol. 52, no. 12, pp. 2134-2142, 2004.

[3] A. Assalini and A. M. Tonello, "Improved nyquist pulses," IEEE Communications Letters, vol. 8, no. 2, pp. 87-89, 2004.

[4] P. Sandeep, S. Chandan, and A. K. Chaturvedi, "ISI-free pulses with reduced sensitivity to timing errors," IEEE Communications Letters, vol. 9, no. 4, pp. 292-294, 2005.

[5] S. Chandan, P. Sandeep, and A. K. Chaturvedi, "A family of ISIfree polynomial pulses," IEEE Communications Letters, vol. 9, no. 6, pp. 496-498, 2005.

[6] S. D. Assimonis, M. Matthaiou, and G. K. Karagiannidis, "Two-parameter Nyquist pulses with better performance," IEEE Communications Letters, vol. 12, no. 11, pp. 807-809, 2008.

[7] S. D. Assimonis, M. Matthaiou, G. K. Karagiannidis, and J. A. Nossek, "Improved parametric families of intersymbol interference-free Nyquist pulses using inner and outer functions," IET Signal Processing, vol. 5, no. 2, pp. 157-163, 2011.

[8] M. Mohri and M. Hamamura, "ISI-free power roll-off pulse," IEICE Transactions on Fundamentals of Electronics, Communications and Computer Sciences, vol. E92-A, no. 10, pp. 24952497, 2009.

[9] N. D. Alexandru and A. L. O. Balan, "Improved nyquist filters with piece-wise parabolic frequency characteristics," IEEE Communications Letters, vol. 15, no. 5, pp. 473-475, 2011.

[10] A. L. Balan and N. D. Alexandru, "Two improved nyquist filters with piece-wise rectangular-polynomial frequency characteristics," AEÜ-International Journal of Electronics and Communications, vol. 66, no. 11, pp. 880-883, 2012.

[11] N. D. Alexandru and A. L. Balan, "ISI-free pulses produced by improved Nyquist filter with piece-wise linear characteristic," Electronics Letters, vol. 47, no. 4, pp. 256-257, 2011.

[12] N. C. Beaulieu, "The evaluation of error probabilities for intersymbol and cochannel interference," IEEE Transactions on Communications, vol. 39, no. 12, pp. 1740-1749, 1991. 


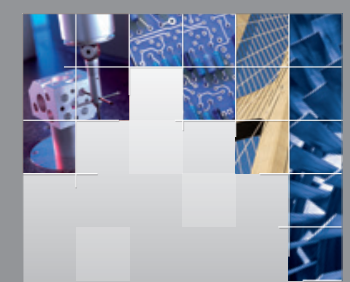

\section{Enfincering}
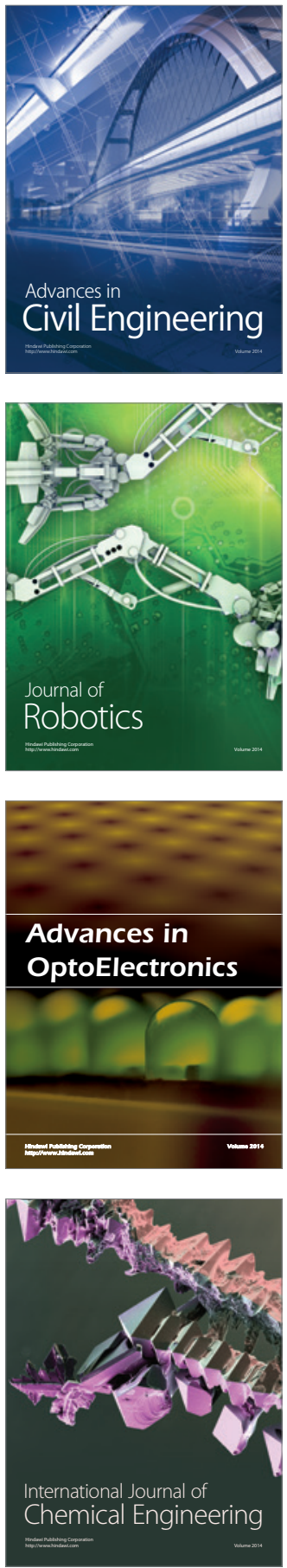

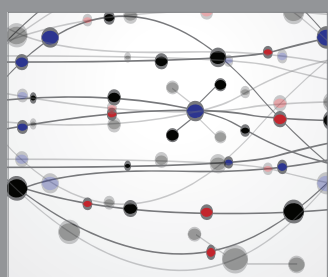

The Scientific World Journal

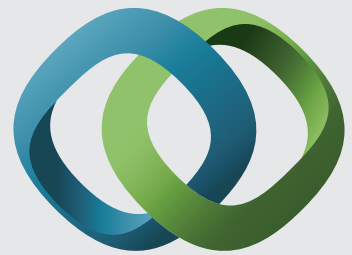

\section{Hindawi}

Submit your manuscripts at

https://www.hindawi.com
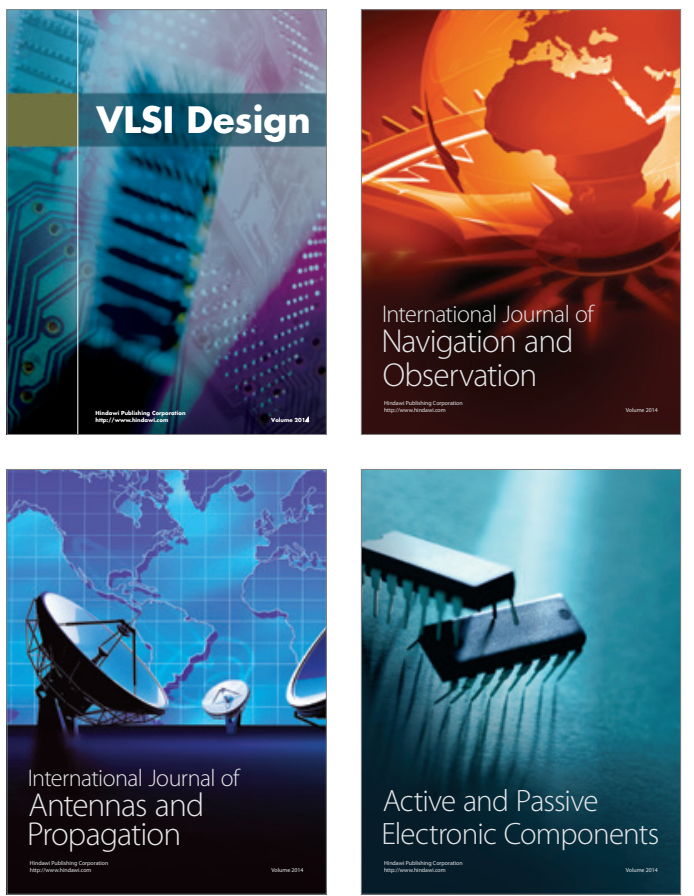
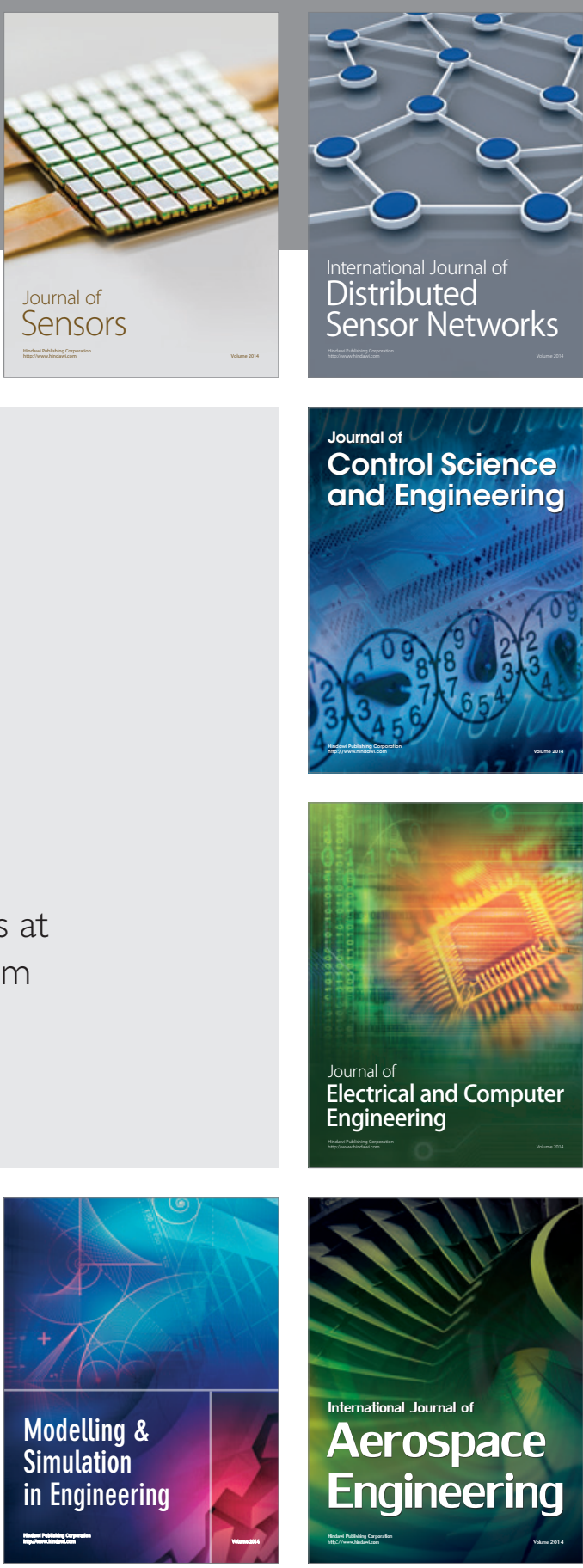

International Journal of

Distributed

Sensor Networks

$-$

Joumal of

Control Science

and Engineering
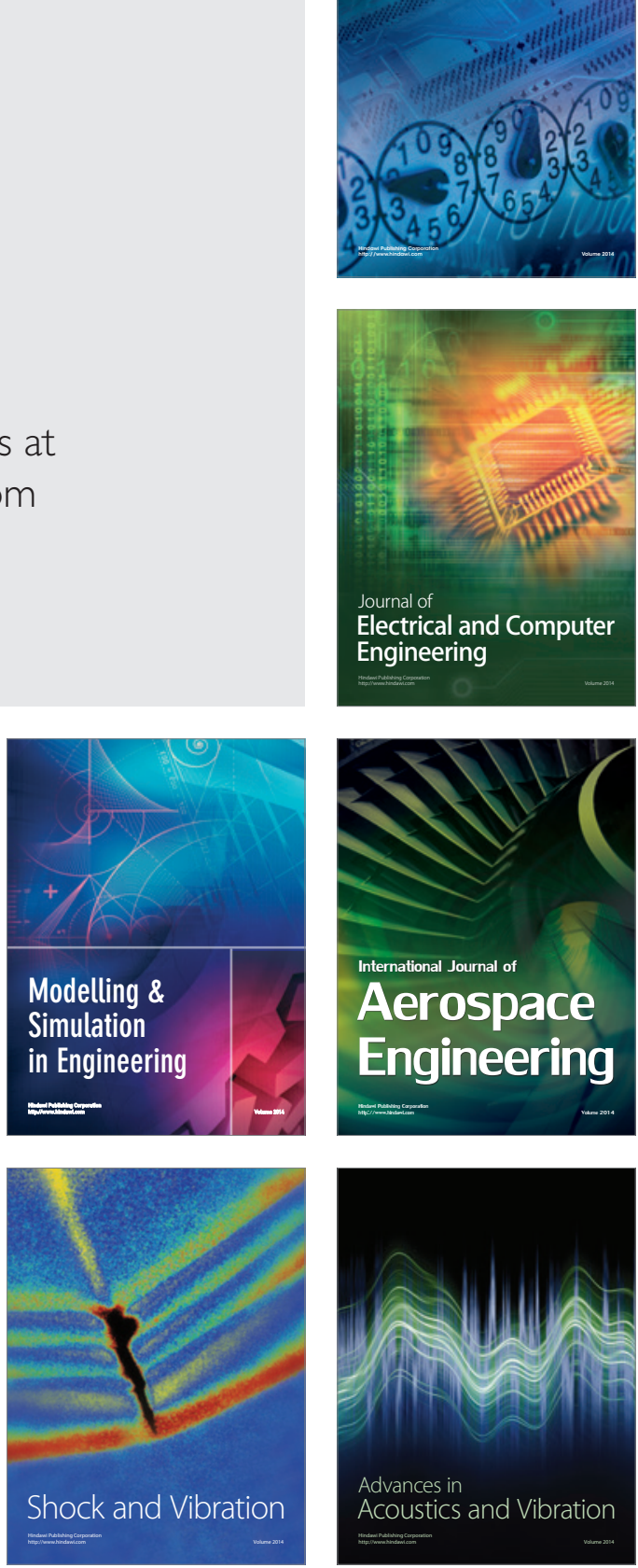